\title{
Descompresión lumbar en pacientes con enfermedad de Parkinson: serie de casos tratados con cirugía mínimamente invasiva
}

\author{
Nicolás Coombes, Eduardo Galaretto, Juan Pablo Guyot, Norberto Fernández, Cristian Fuster \\ Axial Grupo Médico, Ciudad Autónoma de Buenos Aires, Argentina
}

\begin{abstract}
RESUMEN
Introducción: La enfermedad de Parkinson afecta principalmente a personas >65 años. El cuadro degenerativo lumbar en el contexto de un canal estrecho sumado a la alteración neuromuscular y la mala calidad ósea, favorece al desequilibrio sagital y coronal. Los pacientes que necesitan cirugía tienen altas tasas de revisión y reoperación con técnicas instrumentadas y no instrumentadas. El objetivo de este estudio fue evaluar la técnica quirúrgica de descompresión mínimamente invasiva en pacientes con enfermedad de Parkinson y dolor radicular o claudicación neurogénica en los miembros inferiores. Materiales y Métodos: Se evaluó a pacientes con diagnóstico de canal lumbar estrecho y enfermedad de Parkinson tratados quirúrgicamente. El procedimiento se indicó por síntomas de canal estrecho o estenosis foraminal de causa degenerativa. Todos fueron tratados con una cirugía descompresiva a través de una hemilaminectomía mínimamente invasiva mediante retracción tubular y asistencia con microscopia. Resultados: De enero de 2015 a diciembre de 2017, se trató a 6 pacientes con enfermedad de Parkinson y canal lumbar estrecho. Se descomprimieron 12 niveles en total, todos estuvieron internados $<24 \mathrm{~h}$. No se necesitaron transfusiones, no hubo complicaciones asociadas, el tiempo promedio de cirugía fue de 120 minutos. El seguimiento mínimo fue de 12 meses, los síntomas no reaparecieron. Conclusiones: La elevada tasa de complicaciones en pacientes operados con enfermedad de Parkinson y la disminución de las complicaciones en este estudio sugieren que la cirugía mínimamente invasiva de columna lumbar debería ser una alternativa quirúrgica en estos pacientes.
\end{abstract}

Palabras clave: Estenosis de canal; enfermedad de Parkinson; descompresión mínimamente invasiva.

Nivel de Evidencia: IV

Lumbar Decompression in Patients with Parkinson's Disease: Series of Cases Treated with Minimally Invasive Surgery

\begin{abstract}
Introduction: Parkinson's disease mainly affects patients aged 65 and older. The degenerative condition at the lumbar spine in the context of a narrow canal added to the neuromuscular disorder and poor bone quality favors sagittal and coronal imbalance. Patients who need surgery have high rates of revision and reoperation with instrumented and non-instrumented techniques. The objective of this study was to evaluate the minimally invasive surgical decompression technique in patients with Parkinson's disease and radicular pain or neurogenic claudication in the lower limbs. Materials and Methods: Surgically treated patients with a diagnosis of lumbar spinal stenosis and Parkinson's disease were evaluated. The indication for the procedure was due to symptoms of the lumbar or foraminal stenosis of degenerative cause. All the patients were treated with decompressive surgery through a minimally invasive hemilaminectomy using tubular retraction and assistance with microscopy. Results: From January 2015 to December 2017, 6 patients with Parkinson's disease and lumbar spinal stenosis were treated. A total of 12 levels were decompressed, all patients were hospitalized for less than 24 hours. They did not require a transfusion nor suffered associated complications, with an average surgery time of 120 minutes. All with a minimum follow-up of 12 months and no relapse of the symptoms. Conclusion: The high rate of complications in patients with Parkinson's disease, in contrast to the low rate of complications in the present study, suggests that minimally invasive surgery of the lumbar spine should be a surgical alternative in these patients.
\end{abstract}

Key words: Spinal stenosis; Parkinson's disease; minimally invasive decompression.

Level of Evidence: IV 


\section{INTRODUCCIÓN}

La enfermedad de Parkinson (EP) es un trastorno neurodegenerativo que afecta principalmente a adultos con una incidencia calculada de entre el $1 \%$ y el $4 \%$ de la población $>60$ años. ${ }^{1}$ Se caracteriza por una serie de manifestaciones de tipo motoras, como temblor, bradicinesias, rigidez muscular, así como también alteraciones en la marcha y la postura. ${ }^{2}$

Se han descrito múltiples alteraciones espinales principalmente relacionadas con trastornos del equilibrio tanto en el plano coronal como en el sagital (Figuras 1-3).

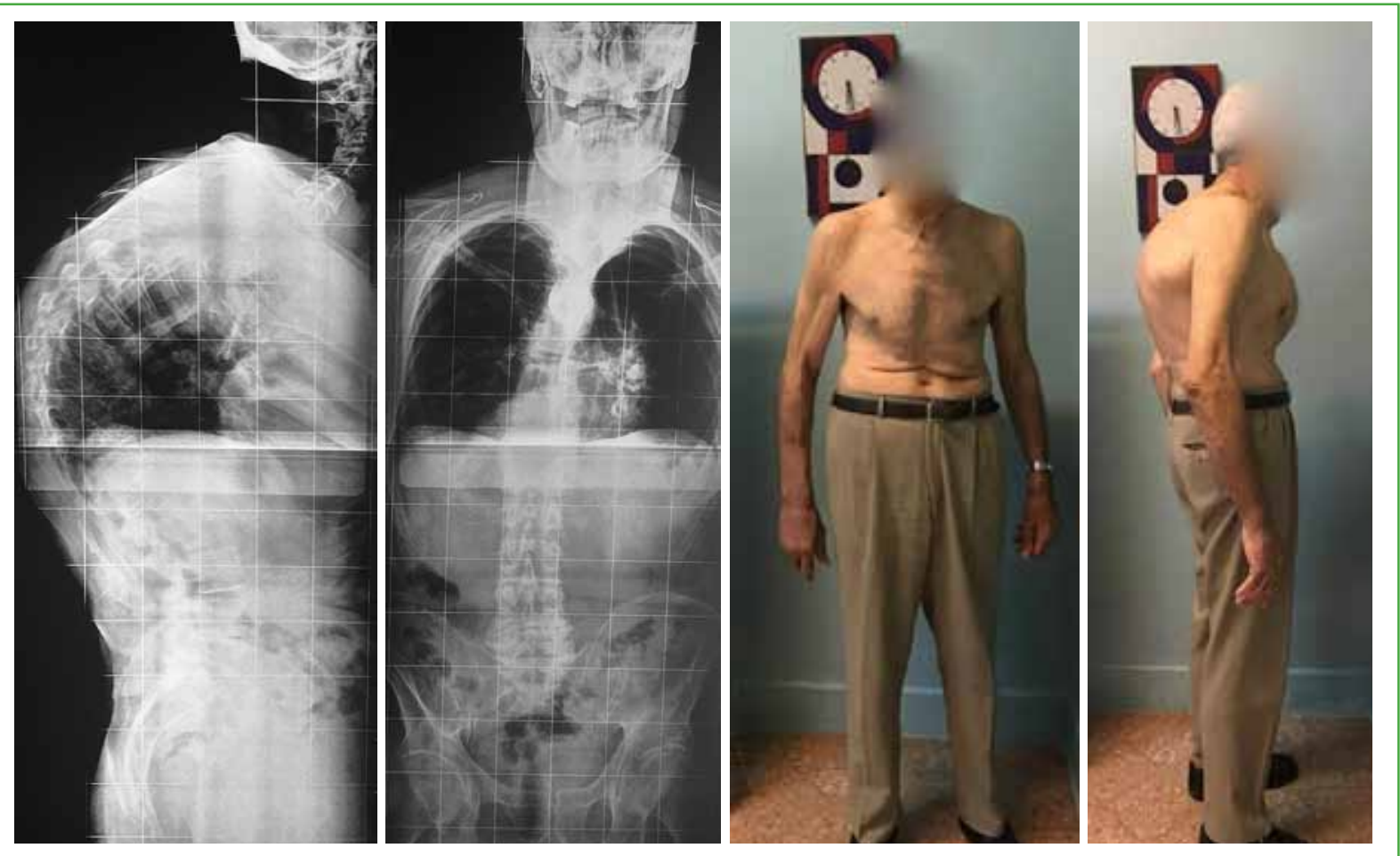

Figura 1. Aspecto clínico y radiográfico de un paciente con desequilibrio coronal y sagital que sufre enfermedad de Parkinson.

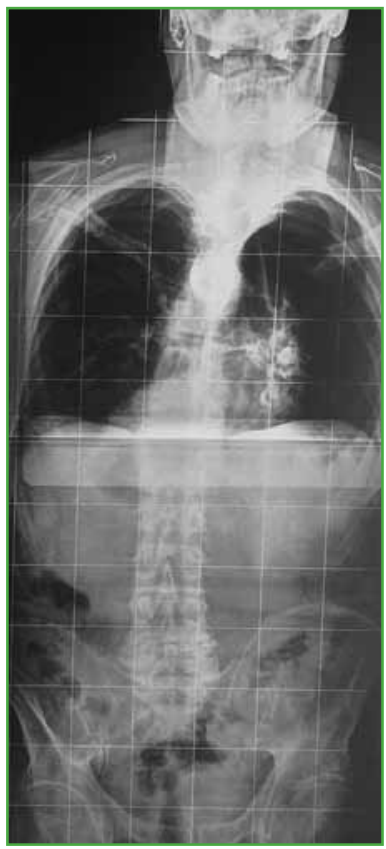

Figura 2. Síndrome de

Pisa en un paciente con enfermedad de Parkinson.

Figura 3. Alteración del plano sagital en la imagen lateral del espinograma.

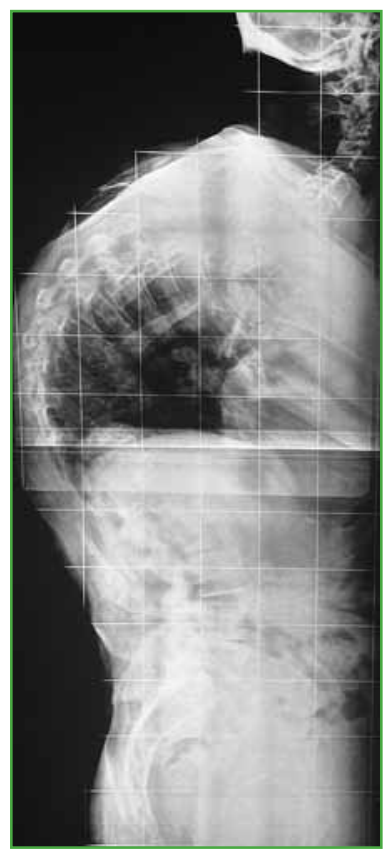


En este tipo de pacientes, las deformidades posturales asociadas están relacionadas con el aumento del tono de los músculos axiales y de las extremidades acompañado de la disminución progresiva de la propiocepción neuronal. ${ }^{3,4} \mathrm{El} \mathrm{3-12,9 \%}$ tiene camptocormia, se trata de una postura anormal con una marcada flexión de la columna toracolumbar que se incrementa con el tiempo y la fatiga durante el día y al caminar. Es una insuficiencia postural progresiva que disminuye en decúbito supino o en posición sentada, o se corrige cuando el paciente con EP se apoya contra una pared. Este cuadro se asocia no solo con la EP, sino que también puede observarse en pacientes con enfermedades por otras causas, como miopatías, miositis, atrofia multisistémica y trastorno de conversión. Por lo general, no hay anormalidades radiográficas o cambios estructurales que se refieran a ese tipo de disfunción neuromuscular. ${ }^{5}$

Al mismo tiempo, la EP genera una alteración del metabolismo fosfocálcico y la consecuente osteoporosis, esto aumenta las posibilidades de fracturas por aplastamientos vertebrales en forma espontánea y el consiguiente agravamiento de la deformidad. ${ }^{4}$

Por otra parte, al tratarse de una población generalmente adulta, los cambios degenerativos y la estenosis resultante del conducto vertebral alteran más la calidad de vida de estos pacientes.

En estos casos, la solución quirúrgica se considera un procedimiento muy demandante por su alta tasa de complicaciones y la frecuente necesidad de revisión quirúrgica. ${ }^{5,6}$

Se han logrado muy buenos resultados con la cirugía descompresiva mínimamente invasiva de la columna lumbar en pacientes con síntomas secundarios a la estenosis del conducto espinal y se indica, cada vez más, principalmente a pacientes con muchas comorbilidades. ${ }^{7}$

El objetivo de esta publicación es comunicar los resultados preliminares de una técnica quirúrgica descompresiva mínimamente invasiva de la columna lumbar en pacientes con canal estrecho y EP.

\section{MATERIALES Y MÉTODOS}

Se llevó a cabo un estudio retrospectivo en una serie de pacientes con diagnóstico de EP y canal lumbar estrecho asociado, que fueron sometidos a una descompresión mínimamente invasiva de la columna lumbar entre enero de 2015 y diciembre de 2017. Se analizó el síntoma predominante definiéndolo como síntomas de claudicación neurológica o signos radiculares. Se estudiaron los signos de déficit motor o sensitivo, así como también las deformidades posturales asociadas (camptocormia, torre de Pisa, anterocolis).

Se incluyó a pacientes con enfermedad, al menos, en estadio 3 según la clasificación de Hoehn-Yahr. ${ }^{8}$

Los pacientes fueron tratados como medida inicial con terapias de físicas de rehabilitación y, cuando la respuesta fue escasa, se realizaron bloqueos selectivos peridurales o radiculares orientados bajo tomografía computarizada según el síntoma predominante.

Todos fueron estudiados con radiografías estáticas y dinámicas, espinograma, tomografía computarizada y resonancia magnética.

La decisión de indicar la cirugía se tomó en forma multidisciplinaria, principalmente junto con el neurólogo tratante.

Se incluyeron pacientes con diagnóstico de EP y un canal lumbar estrecho, y cuyo síntoma predominante estaba dado por signos neurológicos de claudicación en la marcha o síntomas radiculares (cruralgia o ciática) y que respondían positivamente a los bloqueos selectivos bajo tomografía computarizada.

Se excluyó a aquellos cuyo síntoma principal era la lumbalgia o que tenían antecedentes de cirugía de columna, signos marcados de inestabilidad o deformidad segmentaria más inestabilidad y a quienes no respondían al tratamiento con bloqueos.

La técnica quirúrgica elegida fue una hemilaminectomía descompresiva mínimamente invasiva a nivel lumbar. Todos los procedimientos estuvieron a cargo del mismo equipo quirúrgico. Para la retracción, se utilizó un sistema tubular progresivo de fijación autoestática (Metrx,-Medtronic Inc.), los diámetros tubulares variaron de 18 a 22 mm. El campo se amplificó e iluminó con un microscopio OPMI Pentero 800 (Zeiss). Se registraron la duración del procedimiento, el tiempo promedio de internación y las complicaciones durante el procedimiento y en el posoperatorio.

Se evaluó el dolor en los miembros inferiores mediante la escala analógica visual (rango 1-10) antes de la cirugía, en el posoperatorio inmediato y al año de la intervención. La satisfacción del paciente se determinó con la escala de Weiner y los criterios de MacNab modificados al año de la cirugía. Todos tuvieron un seguimiento mínimo de 12 meses (promedio 24 meses, toda la serie). 


\section{RESULTADOS}

Se incluyó a seis pacientes (5 hombres y 1 mujer) con una edad promedio de 70.5 años (rango 65-78) antes de la cirugía, y una EP grados 3 y 4 de la Escala de Hoehn-Yahr para gravedad de la EP.

En los estudios por imágenes, el hallazgo más común fue la estenosis del conducto lumbar, principalmente a nivel L2-L3, L3-L4 y L4-L5.

Todos estuvieron internados menos de $24 \mathrm{~h}$. La cantidad total de segmentos tratados fue 12 (media 2 niveles por paciente), el segmento más frecuente fue L3/L4 $(41,6 \%, \mathrm{n}=5)$ seguido de L4/L5 $(33,3 \%, \mathrm{n}=4)$ y L5/S1 $(25 \%$, $\mathrm{n}=3$ ). Los datos demográficos se resumen en la Tabla 1. El tiempo operatorio promedio fue de 120 min (rango 103-140).

\begin{tabular}{|c|c|}
\hline Número de pacientes & 6 \\
\hline Sexo Femenino/Masculino & $(1-5)$ \\
\hline Edad promedio & 70,5 \\
\hline $\begin{array}{l}\text { Síntomas } \\
\text { Ciática } \\
\text { Cruralgia } \\
\text { Claudicación }\end{array}$ & $\begin{array}{l}(4-6) \\
(3-6) \\
(3-6)\end{array}$ \\
\hline Bloqueo farmacológico & 6 \\
\hline $\begin{array}{l}\text { Niveles afectados } \\
\text { L2-L3 } \\
\text { L3-L4 } \\
\text { L4-L5 }\end{array}$ & $\begin{array}{l}12 \\
3 \\
5 \\
4\end{array}$ \\
\hline
\end{tabular}

Cuando se realizó una descompresión de hasta dos niveles se usó un solo abordaje $(n=5)$ y solo se recurrió a dos abordajes en un paciente con descompresión de tres niveles $(\mathrm{n}=1)$.

No fue necesario administrar transfusiones y no se registraron complicaciones posoperatorias, como infección, reoperación por recidiva de los síntomas o lesiones durales. El cuadro sintomatológico preoperatorio mejoró en todos los pacientes, esto influyó en la mejoría de la calidad de vida sin interferir en la evolución natural de la enfermedad. Al año de seguimiento, no había signos de inestabilidad en los segmentos tratados, que fueron evaluados mediante radiografías en flexión y extensión.

El puntaje promedio en la escala analógica visual para dolor en los miembros inferiores fue de 8,60, mejoró a 2,6 en el posoperatorio inmediato y llegó a 2,8 al año de la cirugía (Figura 4).

El 83,3\% (n = 5) de los pacientes percibió el tratamiento quirúrgico como muy exitoso o bastante exitoso, según el cuestionario de Weiner, y lo recomendaría a otro individuo que lo requiera. Según la escala modificada de Mac$\mathrm{Nab}$, cinco de los seis pacientes consideraron los resultados quirúrgicos como buenos o excelentes y uno, como regulares (Tabla 2). 


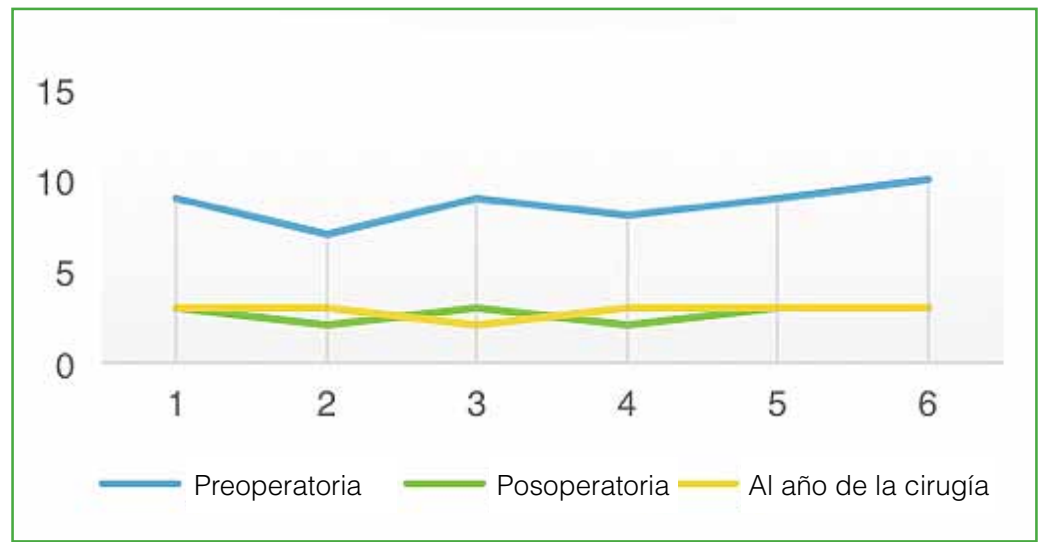

Figura 4. Valores de la escala analógica visual.

Tabla 2. Resultados clínicos

\begin{tabular}{|l|l|}
\hline Datos clínicos & \\
\hline $\begin{array}{l}\text { MacNab modificado } \\
\text { Excelente } \\
\text { Bueno } \\
\text { Regular }\end{array}$ & 3 \\
Malo & 2 \\
\hline Weiner & 1 \\
\hline Mucho éxito & \\
Bastante \\
No mucho \\
Fracaso \\
Peor que antes \\
\end{tabular}

\section{DISCUSIÓN}

En la bibliografía actual, no hay consenso sobre el manejo quirúrgico de la patología espinal en pacientes con EP, incluso es escasa y no hay un claro acuerdo.,9-12

Las complicaciones biomecánicas relacionadas con la instrumentación son las responsables de las altas tasas de revisión, fundamentalmente seudoartrosis, infecciones, aflojamiento del implante y pérdida de la corrección con deformidad progresiva. ${ }^{1,3,7,9,13-15}$

No se han publicado artículos que recomienden la descompresión simple en pacientes con EP. En una serie de seis pacientes, Babat y cols. informan que, luego de descompresiones abiertas con laminectomía, todos tuvieron una mala evolución antes del año de la cirugía. Los pacientes de esta serie habían sido operados, porque tenían un canal lumbar estrecho, cinco fueron sometidos a descompresión, todos desarrollaron inestabilidad y requirieron fusión. El paciente 6 de la serie sometido a descompresión y fusión no instrumentada evolucionó con inestabilidad y seudoartrosis.

En estos pacientes, la activación de factores de compensación lumbopélvicos está disminuida; por lo tanto, la retroversión pélvica, la disminución de la cifosis torácica y la flexión de las rodillas no generan una pérdida de la lordosis lumbar., ${ }^{9}$ Algunos autores han descrito este cambio morfológico de la pelvis como cifosis pélvica. ${ }^{13,17}$ Hay una tendencia a inclinarse hacia adelante, incluida la pelvis, probablemente debido a la degeneración muscular. Wunderlich y cols. han descrito casos en los que la deformidad desarrollada símil camptocormia se relacionaba 
con un proceso de características histopatológicas de miositis a nivel paravertebral; la resonancia magnética mostraba hiperintensidad y edema en la región paravertebral; la respuesta a la corticoterapia fue buena. ${ }^{18}$

En esta serie, no hubo pacientes con alteraciones posturales como las descritas en la bibliografía.

La serie más grande de casos con EP publicada incluye 48 pacientes sometidos a fusiones largas tratados en siete centros, durante 30 años, en Francia. Veinte de estos 48 pacientes fueron sometidos a cirugías de revisión. A pesar de la alta tasa de complicaciones y que solo un tercio de la muestra obtuvo buenos resultados, el $78 \%$ de la población estaba satisfecha con el resultado final..$^{4,10}$

Las tasas de revisión varían del $60 \%$ al $86 \%$, y las principales causas de revisión son la fatiga del implante, el pull-out de los tornillos, la degeneración del segmento adyacente y la infección de la herida quirúrgica. ${ }^{1,3,11}$

En nuestra serie, no hubo complicaciones relacionadas con infección de la herida quirúrgica, algunas series publicadas reportan una tasa de infección que oscila entre el $14 \%$ y el $17 \% .^{1,14}$

La indicación de cirugía parece no estar relacionada con el trastorno neurológico, parece estar más relacionada con el compromiso que sufren estos pacientes en su calidad de vida diaria y las dificultades para relacionarse en su entorno social, el tratamiento quirúrgico intenta lograr la independencia del paciente. ${ }^{4,11}$

No hemos encontrado publicaciones sobre la descompresión del canal lumbar mediante técnicas mínimamente invasivas en pacientes con EP. La mayoría describe resultados asociados a tratamientos quirúrgicos abiertos, correcciones con instrumentaciones y, en menor medida, descompresiones abiertas sin instrumentación, ninguna de estas opciones está exenta de complicaciones. ${ }^{1,3,4,9,13}$

Pensamos que se trata de una opción terapéutica válida teniendo en cuenta que estos pacientes están limitados e imposibilitados, tienen muchos síntomas y una enfermedad progresiva; reanudar sus actividades y recuperar la independencia representan un muy buen resultado clínico, con un daño mínimo sobre la musculatura posterior mediante la técnica mínimamente invasiva.

El elevado riesgo de reoperación y complicaciones se atribuye a una combinación del grave trastorno neuromuscular, la mala calidad ósea y a la cifosis del segmento adyacente que es el mecanismo más común de un resultado fallido..$^{11,14,15}$

En esta serie, la principal ventaja fue que, pese a ser un reporte preliminar de casos, los pacientes evolucionaron, de manera favorable, sin complicaciones asociadas, con una mejoría significativa del dolor posoperatorio, sin agravamiento de los síntomas ni signos de inestabilidad posoperatoria al año de seguimiento.

La principal limitación es que se trata de una serie pequeña, con un tiempo promedio de valoración posoperatoria de 24 meses. Teniendo en cuenta ciertas características de estos pacientes, como la alteración del metabolismo fosfocálcico y el trastorno neuromuscular asociado, podría ser útil un seguimiento más prolongado para evaluar el comportamiento de los segmentos descomprimidos.

\section{CONCLUSIÓN}

Frente a la complejidad que ofrecen los pacientes con EP y canal lumbar estrecho, las opciones terapéuticas actuales incluyen la cirugía de fusión y la descompresión abierta con una tasa alta de morbilidad y revisión. En este sentido, ofrecer una opción terapéutica mínimamente invasiva podría ser muy favorable para evitar las complicaciones mecánicas relacionadas con la instrumentación, disminuir las complicaciones y el tiempo de internación

Conflicto de intereses: Los autores no declaran conflictos de intereses.

ORCID de E. Galaretto: https://orcid.org/0000-0003-4121-8228 ORCID de J. P. Guyot: https://orcid.org/0000-0001-6524-4426
ORCID de N. Fernández: https://orcid.org/0000-0002-5250-5399 ORCID de C. Fuster: https://orcid.org/0000-0002-5293-0846 


\section{BIBLIOGRAFÍA}

1. Babat LB, McLain RF, Bingaman W, Kalfas I, Young P, Rufo- Smith C. Spinal surgery in patients with Parkinson's dis- ease: construct failure and progressive deformity. Spine (Phila Pa 1976) 2004;29:2006-12. https://doi.org/10.1097/01.brs.0000138306.02425.21

2. Boonstra TA, van der Kooij H, Munneke M, Bloem BR. Gait disorders and balance disturbances in Parkinson's disease: clinical update and pathophysiology. Curr Opin Neurol 2008;21:461-71. https://doi.org/10.1097/WCO.0b013e328305bdaf

3. Bourghli A, Guerin P, Vital JM, Aurouer N, Luc S, Gille O, et al. Posterior spinal fusion from T2 to the sacrum for the management of major deformities in patients with Parkinson disease: a retrospective review with analysis of complications. J Spinal Disord Tech 2012;25:E53-E60. https://doi.org/10.1097/BSD.0b013e3182496670

4. Melamed E, Djaldetti R. Camptocormia in Parkinson's disease. J Neurol 2006;253(Suppl 7):VII14-VII16. https://doi.org/10.1007/s00415-006-7004-5

5. Choi S, Kang K, Lee S, Lee S, Park M, Kim B, et al. The association of musculoskeletal pain with bone mineral density in patients with Parkinson's disease. J Neurol Sci 2015;357:e261-e262. https://doi.org/10.1159/000455009

6. Djaldetti R, Mosberg-Galili R, Sroka H, Merims D, Melamed E. Camptocormia (bent spine) in patients with Parkinson's disease - characterization and possible pathogenesis of an unusual phenomenon. Mov Disord 1999;14(3):443-7. https://doi.org/10.1002/1531-8257(199905)14:3<443::aid-mds1009>3.0.co;2-g

7. Mayer HM. Microsurgical decompression of acquired (degenerative) central and lateral spinal canal stenosis. En: Mayer HM (ed.). Minimally invasive spine surgery. Berlin, Germany: Springer; 2000:105-16.

8. Grimes JD, Hassan MN, Trent G, Halle D, Armstrong GW. Clinical and radiographic features of scoliosis in Parkinson's disease. Adv Neurol 1987;45:353-5. PMID: 3825710

9. Upadhyaya CD, Starr PA, Mummaneni PV. Spinal deformity and Parkinson disease: a treatment algorithm. Neurosurg Focus 2010;28:E5. https://doi.org/10.3171/2010.1.FOCUS09288

10. Koller H, Acosta F, Zenner J, Ferraris L, Hitzl W, Meier O, et al. Spinal surgery in patients with Parkinson's disease: experiences with the challenges posed by sagittal imbalance and the Parkinson's spine. Eur Spine J 2010;19(10):1785-94. https://doi.org/10.1007/s00586-010-1405-y

11. Moon SH, Lee HM, Chun HJ, Kang KT, Kim HS, Park JO, et al. Surgical outcome of lumbar fusion surgery in patients with Parkinson disease. J Spinal Disord Tech 2012;25:351-5. https://doi.org/10.1097/BSD.0b013e318224a625

12. Oh JK, Smith JS, Sharey CI, Lafage V, Schwab F, Ames CP, et al. Sagittal spinopelvic malalignment in Parkinson disease: prevalence and associations with disease severity. Spine (Phila Pa 1976) 2014;39:E833-E841. https://doi.org/10.1097/BRS.0000000000000366

13. Galbusera F, Bassani T, Stucovitz E, Martini C, Maryem-Fama I, Berjano P, et al. Surgical treatment of spinal disorders in Parkinson's disease. Eur Spine J 2018 ;27(Suppl 1):101-8. https://doi.org/10.1007/s00586-018-5499-y

14. Kim YJ, Bridwell KH, Lenke LG, Rhim S, Cheh G. Pseudarthrosis in long adult spinal deformity instrumentation and fusion to the sacrum: prevalence and risk factor analysis of 144 cases. Spine 2006;31:2329-36. https://doi.org/10.1097/01.brs.0000238968.82799.d9

15. Tsuchiya K, Bridwell KH, Kuklo TR, Lenke LG, Baldus C. Minimum 5-year analysis of L5-S1 fusion using sacropelvic fixation (bilateral S1 and iliac screws) for spinal deformity. Spine (Phila Pa 1976) 2006;31(3):303-8. https://doi.org/10.1097/01.brs.0000197193.81296.f1

16. Watanabe K, Hirano T, Katsumi K, Ohashi M, Shoji H, Hasegawa K, et al. Characteristics of spinopelvic alignment in Parkinson's disease: Comparison with adult spinal deformity. J Orthop Sci 2017;22(1):16-21. https//doi.org/10.1016/j.jos.2016.09.013

17. Lamartina C, Berjano P.Classification of sagittal imbalance based on spinal alignment and compensatory mechanisms. Eur Spine J 2014;23:1177-89. https://doi.org/10.1007/s00586-014-3227-9

18. Wunderlich S, Csoti I, Reiners K, Günthner-Lengsfeld T, Schneider C, Becker G, et al. Camptocormia in Parkinson's disease mimicked by focal myositis of the paraspinal muscles. Mov Disord 2002;17(3):598-600. https://doi.org/10.1002/mds.10110 